\title{
Correlação das alterações osteomioarticulares e dor em idosos de Morrinhos(CE)
}

\author{
Giselle Santos Meneses*, Renata Oliveira Leorne", Samara Sousa Vasconcelos Gouveia", \\ Guilherme Pertinni de Morais Gouveia**
}

\section{Resumo}

O envelhecimento é caracterizado como um processo dinâmico e progressivo a todos os seres vivos, proporcionando a cada um que envelhece características próprias, decorrentes de modificações tanto morfológicas, funcionais e bioquímicas, que irão interferir na capacidade de adaptação do indivíduo ao ambiente em que vive, ocasionando limitações funcionais típicas e inevitáveis. Com o aumento da população idosa e, com isso, o seu envelhecimento, há algumas dificuldades a serem enfrentadas, logo, torna-se importante analisar a prevalência de alterações osteomioarticulares, bem como as características presentes nessa população. O objetivo a que nos propomos é correlacionar as alterações osteoarticulares e dor em idosos de Morrinhos (CE). Como métodos de análise partimos de um estudo transversal, descritivo e analítico, de abordagem quantitativa, realizado na cidade de Morrinhos. A pesquisa realizou-se com 270 idosos residentes no município. Foi utilizada a Escala Visual Analógica (EVA) e o Questionário Nórdico de Sintomas Osteomusculares. Os dados foram analisados com o teste de correlação de Spearman e teste $\mathrm{T}$ para amostras independentes, considerando-se significância estatística de $p \leq 0,05$. Os resultados obtidos na pesquisa, dentre as alterações clínicas encontradas, pode-se destacar que, $77,8 \%$ dos idosos afirmaram apresentar sintomas osteoarticulares; quanto à caracterização da dor, 86,5\% mencionaram sentir dor em alguma região do corpo, uma vez que a dor articular é a mais presente $(81,8 \%)$. Pode-se afirmar ainda que, a presença de alterações osteoarticulares foi o fator mais relacionado à ocorrência de dor nos idosos. Por fim, concluimos que há alta prevalência de sintomas osteomioarticulares na população idosa do município de Morrinhos e esses sintomas geralmente são acompanhadas por desconfortos álgicos.

Palavras-chave: Idosos. Sistema musculoesquelético. Dor.

* Graduadas em Fisioterapia pelas Faculdades INTA.

* Fisioterapeuta. Mestre em Saúde Pública pela Universidade Federal do Ceará. Docente do curso de Fisioterapia da Universidade Federal do Piauí.

**** Orientador. Fisioterapeuta. Mestre em Saúde Pública pela Universidade Federal do Ceará. Doutorando em Cirurgia pela Universidade Federal do Ceará. Docente do curso de Fisioterapia da Universidade Federal do Piauí (UFPI). Endereço para correspondência: Universidade Federal do Piauí - Campus Parnaíba, Departamento de Fisioterapia. Av. São Sebastião, 2819, CEP 64202-020, Parnaíba (PI). E-mail: guilherme_pertinni@ yahoo.com.br.

$\rightarrow$ http://dx.doi.org/10.5335/rbceh.2013.3044 


\section{Introdução}

Nos últimos anos, o envelhecimento populacional é considerado um fenômeno mundial, devido ao rápido crescimento da população com mais de sessenta anos (FIEDLER; PERES, 2008a).

O envelhecimento é caracterizado como um processo dinâmico e progressivo a todos os seres vivos, proporcionando a cada um que envelhece características próprias, decorrentes de modificações morfológicas, funcionais e bioquímicas, que irão interferir na capacidade de adaptação do indivíduo ao ambiente em que vive, ocasionando limitações funcionais típicas e inevitáveis do envelhecimento (SCHVEITZER; CLAUDINO, 2010).

De acordo com Santos (2011), quanto maior o envelhecimento populacional, maior será a prevalência de agravos à saúde e incapacidades funcionais associadas.

Em relação às alterações decorrentes do processo de envelhecimento, uma das principais queixas dos idosos segundo Andrade, Pereira e Sousa (2006) e Reis et al. (2008), são as alterações osteomioarticulares, geradas por perdas estruturais e funcionais. Segundo Dellaroza, Pimenta e Matsuo (2007), muitos desses quadros são acompanhados por desconfortos álgicos, visto que a dor crônica é a principal queixa do indivíduo, podendo interferir de modo acentuado na qualidade de vida dos idosos.

Para Dellaroza, Pimenta e Matsuo (2007), o envelhecimento populacional surge como um dos maiores desafios para a saúde na última década. Embora o envelhecimento não seja sinônimo de doença, na maioria das vezes, não é caracterizado como um período saudável e de independência. Ao contrário, depara-se com uma alta incidência de doenças crônico-degenerativas que, muitas vezes, resultam em elevada dependência funcional.

Com o aumento da população idosa e com o envelhecimento dessa, vários problemas tornam-se presentes, logo, torna-se importante analisar a prevalência de alterações osteomioarticulares, bem como, as características presentes nessa população,para com base nessas informações, ser possível detectar os fatores causais, amenizando, assim, as alterações decorrentes do processo de envelhecimento, prevenindo incapacidades e sofrimentos, e visando a melhora da qualidade de vida aos idosos. Acredita-se, que o presente estudo proporcionará reflexões relacionadas ao processo de envelhecimento, assim como, as alterações decorrentes desse processo, pois apesar do crescente interesse em estudos com a população idosa, ainda são escassos no Brasil, sobretudo em municípios de pequeno porte.

A hipótese que se pretende mostrar com este estudo é que as alterações osteoarticulares estejam relacionadas às queixas álgicas, uma vez que o objetivo é correlacionar as alterações osteomioarticulares e dor em idosos de Morrinhos (CE).

\section{Metodologia}

Trata-se de um estudo transversal, descritivo e analítico, de abordagem quantitativa, realizado na cidade de Morrinhos, zona norte do Ceará, no período de novembro a dezembro de 2012.

A amostra foi do tipo probabilística estratificada aleatória retirada da população de idosos residentes no município de Morrinhos. Para cálculo do tamanho amostral, consideraram-se os seguintes 
parâmetros: tamanho da população $(\mathrm{N}=$ 2.181) (GOUVEIA, 2011), prevalência de alteração da funcionalidade inadequada de $30 \%$, erro igual a cinco pontos percentuais e intervalo de confiança de 95\% (IC95\%). O tamanho da amostra obtido ( $\mathrm{n}=180$ ) foi corrigido em 1,2 devido ao efeito do desenho e acrescido $25 \%$ devido às eventuais perdas e/ou recusas, totalizando 275 indivíduos (FIEDLER; PERES, 2008b).

Os critérios de inclusão utilizados foram: residir no município de Morrinhos; ter idade igual ou superior a sessenta anos; ter condições cognitivas e verbais de forma a viabilizar o diálogo, essencial para o preenchimento dos instrumentos de coleta de dados; e, concordar em participar da pesquisa, através da assinatura do termo de consentimento livre e esclarecido. Foram excluídos os idosos que tivessem escore classificatório do Miniexame do Estado Mental abaixo de 19 pontos, pois esse objetiva avaliar o estado cognitivo do idoso (BRASIL, MS, 2006), ou que retirasse seu consentimento.

A coleta de dados foi realizada nas Unidades de Saúde de Morrinhos, convidando cada idoso a comparecer à unidade a que estava adscrito. Os dados foram coletados pela própria pesquisadora, a qual, inicialmente realiza a leitura e a assinatura do Termo de Consentimento Livre e Esclarecido. Em seguida, foi realizada uma avaliação das funções mentais por meio do Miniexame do Estado Mental, um dos critérios de seleção. Após, deu-se a coleta de dados sociodemográficos e clínicos referentes às alterações osteomioarticulares e à dor. Como instrumentos para coleta dos dados, referentes às queixas sintomato- lógicas, foram utilizados a Escala Visual Analógica (EVA), a fim de mensurar a intensidade da dor no paciente e o Questionário Nórdico de Sintomas Osteomusculares desenvolvido com o intuito de padronizar a mensuração de sintomas osteomusculares, facilitando a comparação dos resultados entre pesquisas.

Os dados foram digitados em um banco de dados, utilizando o programa Epi Info (versão 6.04d, Centers for Disease Controland Prevention, EUA). A análise dos dados foi realizada com o software Stata (versão 10; College Station, USA).

A análise inicial foi realizada através da estatística descritiva, incluindo tabulações de acordo com as variáveis selecionadas. A variável "sintomas osteomioarticulares" foi dividida em duas categorias: presença ou ausência de sintomatologia e, posteriormente, aplicado o teste de Correlação de Spearman com a variável "dor muscular ou articular". Para confirmação de tais resultados, foi utilizado o teste $\mathrm{T}$ para amostras independentes, comparando-se a média da EVA entre os grupos que apresentavam e não apresentavam sintomas osteomioarticulares.

Essa pesquisa foi realizada em conformidade com os princípios éticos da resolução CNS 466/12 (BATAGELLO, 2012). Os idosos foram orientados em relação aos objetivos, o procedimento da pesquisa e quanto a garantia do anonimato. Todos assinaram ou identificaram-se com a marca digital o termo de consentimento livre esclarecido (TCL). A pesquisa foi avaliada e obteve parecer favorável do Comitê de Ética em Pesquisa da Universidade Estadual Vale do Acaraú, parecer n. 118.762. 


\section{Resultados e discussão}

\section{Características sociodemográficas}

Participaram do estudo 275 idosos, com idade média de $70 \pm 8$ anos, mediana de 77 anos. A maioria era do sexo feminino $(61,5 \%)$, branca $(36,4 \%)$, casada $(70,5 \%)$ e com nível de escolari- dade fundamental incompleto (44,7\%). A maioria dos participantes é proveniente da zona urbana $(71,3 \%)$, fumante, etilista e praticante de atividade física, com $29,1 \%, 11,6 \%$ e $45,1 \%$, respectivamente, e possuía média de nove filhos. A Tabela 1 apresenta uma descrição detalhada dessas características.

Tabela 1 - Características socioeconômicas e demográficas dos idosos residentes em Morrinhos, no período de julho a outubro de 2012

\begin{tabular}{|c|c|c|}
\hline Variáveis & $\mathrm{n}$ & $\%$ \\
\hline \multicolumn{3}{|l|}{ Sexo } \\
\hline Feminino & 169 & 61,5 \\
\hline Masculino & 106 & 38,5 \\
\hline \multicolumn{3}{|l|}{ Zona de proveniência } \\
\hline Urbana & 196 & 71,3 \\
\hline Rural & 79 & 28,7 \\
\hline \multicolumn{3}{|l|}{ Estado civil } \\
\hline Solteiro & 17 & 6,2 \\
\hline Casado & 194 & 70,5 \\
\hline Divorciado & 5 & 1,8 \\
\hline Desquitado & 6 & 2,2 \\
\hline Viúvo & 52 & 18,9 \\
\hline Outros & 1 & 0,4 \\
\hline \multicolumn{3}{|l|}{ Raça } \\
\hline Branca & 100 & 36,4 \\
\hline Amarela & 74 & 26,9 \\
\hline Parda & 37 & 13,5 \\
\hline Negra & 64 & 23,3 \\
\hline Indígena & 0 & 0,0 \\
\hline \multicolumn{3}{|l|}{ Escolaridade } \\
\hline Analfabeto & 110 & 40,0 \\
\hline Fundamental incompleto & 123 & 44,7 \\
\hline Fundamental completo & 21 & 7,6 \\
\hline Médio incompleto & 4 & 1,5 \\
\hline Médio completo & 11 & 4,0 \\
\hline Superior incompleto & 1 & 0,4 \\
\hline Superior completo & 5 & 1,8 \\
\hline \multicolumn{3}{|l|}{ Fumante } \\
\hline Sim & 80 & 29,1 \\
\hline Não & 195 & 70,9 \\
\hline \multicolumn{3}{|l|}{ Etilista } \\
\hline Sim & 32 & 11,6 \\
\hline Não & 243 & 88,4 \\
\hline \multicolumn{3}{|l|}{ Pratica atividade física } \\
\hline Sim & 124 & 45,1 \\
\hline Não & 151 & 54,9 \\
\hline
\end{tabular}

Fonte: dados da pesquisa. 
A visualização dessa tabela permite, inicialmente, constatar que há um maior percentual de mulheres $(61,5 \%)$, caracterizando o que os autores chamam de "feminização da velhice" (SOUZA; MORAIS; BARTH, 2006). O que pode estar relacionado à maior longevidade dessas em relação aos homens, isso pode ser atribuído à menor exposição a determinados fatores de risco relacionados ao trabalho, tabagismo e uso de álcool, diferenças quanto à atitude em relação a doenças e incapacidades e pela maior cobertura da assistência gineco-obstétrica (PILGER; MENON; MATHIAS, 2011).

Nota-se que há um predomínio dessa população na zona urbana. Dados da Pesquisa Nacional por Amostra de Domicílios (PNAD) que, em 2007, estimaram uma população de sessenta anos ou mais, com aproximadamente 19,9 milhões de pessoas, das quais $82,93 \%$ viviam no meio urbano, evidenciando os resultados obtidos neste trabalho, obtendo uma proporção muito maior de indivíduos idosos nas áreas urbanas $(71,3 \%)$ do que nas rurais.

Em se tratando do estado civil, a maioria referiu ser casada $(70,5 \%)$. Registrou-se ainda, um percentual significativo de idosos viúvos (18,9\%). Esse dado também foi observado no estudo de Celich e Galon (2009), uma vez que a maioria dos idosos que participaram do referido estudo são casados, seguidos pelos viúvos.

Quando analisado o nível de escolaridade dos idosos, observou-se um baixo índice, em que $44,7 \%$ não completaram o ensino fundamental; $7,6 \%$ possuíam o ensino fundamental completo; $4,0 \%$ cursaram o ensino médio completo, e apenas 1,8\% dos idosos possuíam o ensino superior completo.

Os baixos índices de escolaridade são evidenciados ainda em outros estudos como no de Conte, Lopes e Silva (2004) realizado em Marechal Cândido Rondon (PR), revelando que $79,4 \%$ das idosas também não possuíam o ensino fundamental completo; e em Florianópolis (SC), Mazo (2003) detectou que 92\% das idosas não completaram o ensino fundamental. O baixo índice de escolaridade deve-se, principalmente, ao difícil acesso, em décadas passadas, às instituições de ensino, à procedência do meio rural, levando-os a auxiliar no trabalho agrícola para ajudar na renda da família, pois os pais pensavam não haver necessidade de estudo.

Em geral, os dados que caracterizam os idosos participantes deste estudo são semelhantes aos encontrados por outros pesquisadores (DELLAROZA et al., 2008; PANAZZOLO et al., 2007).

Variáveis relacionadas ao perfil clínico dos idosos

Em relação aos sintomas osteoarticulares, 77,8\% dos idosos afirmaram apresentá-los, uma vez que a dor muscular ou articular fez-se presente em 85,5\% dos casos. Quanto aos problemas de saúde associados, as alterações visuais foram os mais prevalentes $(83,3 \%)$, seguidas pela hipertensão arterial $(44,7 \%)$ e diabetes $(18,2 \%)$, conforme a Tabela 2 . 
Tabela 2 - Variáveis relacionadas ao perfil clínico de idosos residentes em Morrinhos, no período de julho a outubro de 2012

\begin{tabular}{lrc}
\hline \multicolumn{1}{c}{ Variáveis } & $\mathrm{n}$ & $\%$ \\
\hline Apresenta sintomas osteoarticulares? & 214 & 77,8 \\
Sim & 61 & 22,2 \\
Não & & \\
Apresenta dor muscular ou articular? & 235 & 85,5 \\
Sim & 40 & 14,5 \\
Não & & \\
Sofreu fratura, traumatismo ou lesão? & 69 & 25,1 \\
Sim & 206 & 74,9 \\
Não & & \\
Já foi submetido à alguma cirurgia? & 117 & 42,5 \\
Sim & 158 & 57,5 \\
Não & & \\
Problemas de saúde que apresenta & 229 & 83,3 \\
Alterações visuais & 123 & 44,7 \\
Hipertensão arterial & 50 & 18,2 \\
Diabetes & 39 & 14,2 \\
Alterações auditivas & 31 & 11,3 \\
Osteoporose & 28 & 10,2 \\
Artrose & 26 & 9,5 \\
Doença respiratória & 21 & 7,6 \\
Depressão & 18 & 6,5 \\
Doença cardíaca & 16 & 5,8 \\
Doença vascular & 16 & $5,4,5$ \\
Labirintite & 13 & 4,7 \\
Deformidade postural & 5 & 1,8 \\
Incontinência & 5 & 0,7 \\
AVE & 23 & \\
Câncer & 103 & \\
Alzheimer & & \\
Outros & 5 & \\
\hline
\end{tabular}

Fonte: dados da pesquisa.

Segundo Andrade, Pereira e Sousa (2006) e Reis et al. (2008), as alterações osteomioarticulares geradas por perdas estruturais e funcionais são uma das principais queixas dos idosos.

Quanto aos problemas de saúde dos participantes, o presente estudo pode ser comparado aos resultados de Lebrão e Duarte (2003), em uma pesquisa realizada no município de São Paulo, em que $70,4 \%$ tinham hipertensão arterial, seguida de diabetes com $14,1 \%$.
Variáveis relacionadas à caracterização da dor

Em relação à análise da EVA, os idosos apresentaram nível leve a moderado de dor, com média da pontuação de 3,42 (土2). Quanto à caracterização da dor, $86,5 \%$ referiram sentir dor em alguma região do corpo, visto ser a dor articular a mais presente (81,8\%). Quanto à localidade da dor, a região lombar foi a mais acometida $(40,4 \%)$, seguida pela região torácica $(38,5 \%)$. Em relação à tempora- 
lidade da dor, $66,5 \%$ dos idosos referiram que a dor está presente há anos e é mais comum no turno da noite $(29,5 \%)$.

Os idosos referiram que a dor amenizava quando permaneciam em repouso $(79,3 \%)$ e que, ao persistir, tomavam algum medicamento oral para analgesia $(63,5 \%)$. Ressaltaram, ainda, que essas dores interferem na sua qualidade de vida, considerada como um razoável incômodo $(46,9 \%)$. Essas variáveis encontram-se detalhadas na Tabela 3.

Tabela 3 - Caracterização de dor nos idosos do município de Morrinhos, no período de julho a outubro de 2012

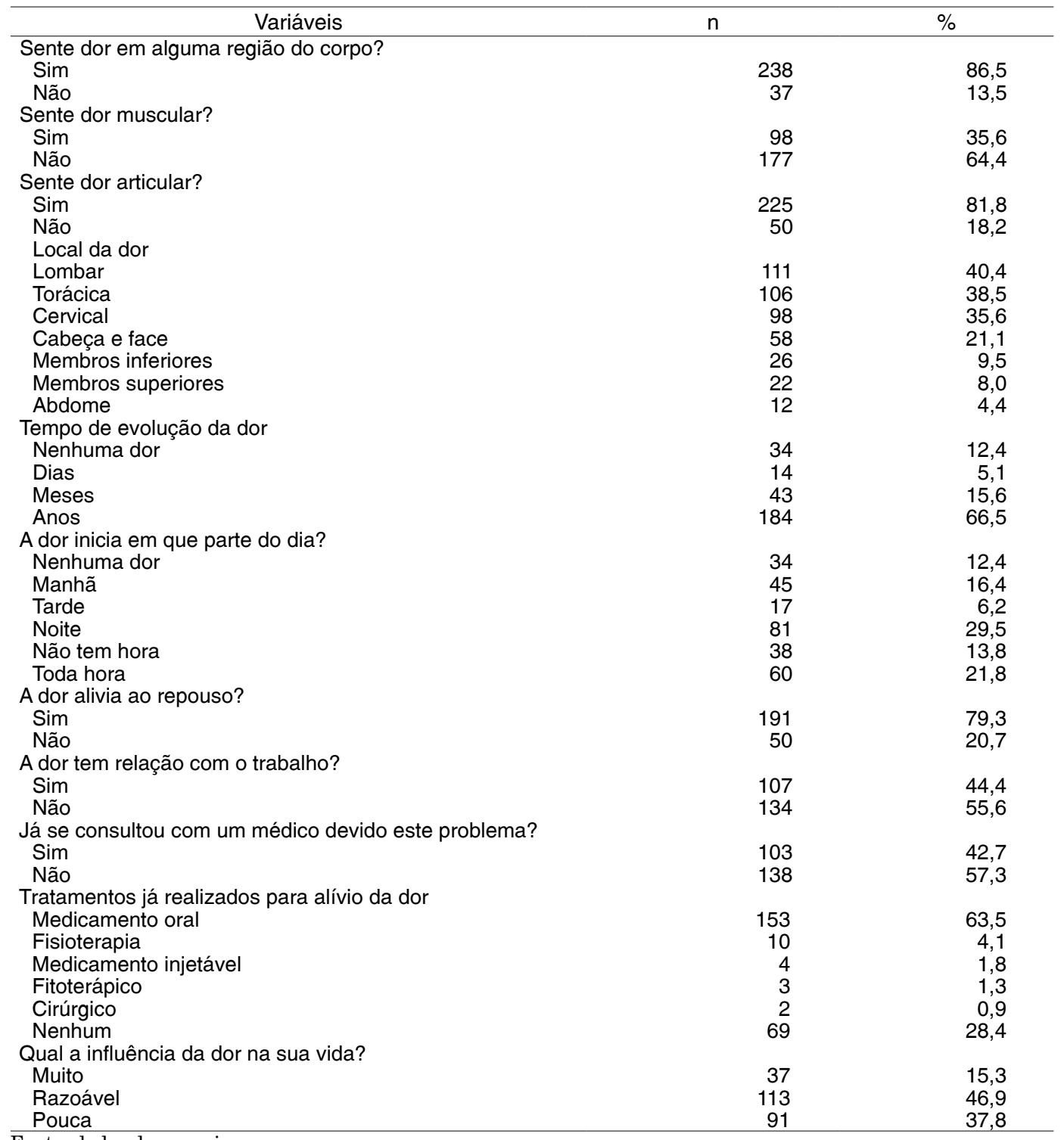

Fonte: dados da pesquisa. 
A prevalência de dor relatada pelos participantes desse estudo foi de 86,5\%, o que corrobora com o estudo de Panazzolo et al. (2007), desenvolvido com amostra de 245 idosos, cujas idades variaram de sessenta a 91 anos, constatando-se um predomínio de dor de $73,9 \%$, o que vem demonstrando uma alta prevalência de dor na população idosa.

Um estudo realizado por Lacerda et al. (2005), em Goiânia, avaliou quarenta idosos atendidos por uma equipe do Programa Saúde da Família (PSF) e identificou que $62,5 \%$ apresentaram dor, confirmando-se, portanto, que a dor é uma condição frequente na população idosa.

Quanto à caracterização da dor, a articular foi a mais presente $(81,8 \%)$, assim nos estudos de Pimenta e Teixeira foram avaliados 990 indivíduos com idade superior a 65 anos, observando-se como localizações mais frequentes de dor as dores articulares (50\%), reforçando os dados aqui apresentados.

Neste estudo, a localização da dor foi relatada com maior frequência na região lombar $(40,4 \%)$, seguida da coluna torácica $(38,5 \%)$, em consonância com os resultados de Celich e Galon (2009) que encontraram dor referida com maior frequência na coluna lombar $(44,4 \%)$, região das pernas $(40,7 \%)$, semelhante ainda aos estudos de Panazzolo et al. (2007), que também encontraram 46,5\% de dor nos membros inferiores entre os 245 idosos participantes de sua pesquisa, seguidos pela coluna lombar $(31,0 \%)$.

$\mathrm{Na}$ vida longeva, a experiência dolorosa pode afetar locais que interferiram na autonomia e na independência, impondo limitação funcional a essa população. A prevalência global de dor, não diminui com a idade e torna-se ainda mais incapacitante, interferindo na realização das atividades da vida diária (GOUVEIA, 2011).

Ao considerar o tempo de duração da dor, $66,5 \%$ dos idosos referiram que a dor está presente há anos, visto ser mais comum no turno da noite em 29,5\%. Tsukimoto et al. (2006) destacam a relevância que tem o tempo de duração da dor e, quanto mais longa sua duração, muitos aspectos da vida podem ser afetados, podendo acarretar distúrbios do sono, depressão e irritabilidade.

Em relação às medidas utilizadas para alívio da dor, $79,3 \%$ dos idosos referiram que ficar em repouso melhora o quadro álgico, desses, $63,5 \%$ também fazem uso de medicamentos orais para analgesia se a dor persistir. A respeito dos métodos analgésicos, Jakobsson (2004) encontrou uma média de três métodos por idoso. Os mais utilizados foram analgésicos $(20 \%)$, descansar $(20 \%)$ e distração (15\%).

Dores intensas podem prejudicar a qualidade de vida das pessoas, levando-as à dependência e à perda da autonomia, redução do convívio social, prejudicando seus relacionamentos e atividades de lazer (GOMES; NASCIMENTO; ARAÚJO, 2007). Segundo dados do estudo, as dores interferem na qualidade de vida, consideradas como um razoável incômodo para $46,9 \%$ dos idosos.

Correlação de sintomas osteoarticulares com dor

Ao correlacionar as variáveis "sintomas osteoarticulares" e "sor muscular ou articular", através do teste de correlação 
de Spearman, obteve-se um coeficiente de correlação de 0,77 , com $\mathrm{p}<0,001$, ou seja, houve correlação forte, estatisticamente significante.

Para confirmar esses resultados, calculou-se a média do escore obtido na EVA entre o grupo que apresentava sintomas osteoarticulares e o que não apresentava, utilizando o teste $\mathrm{T}$ para amostras independentes, obtendo-se 0 resultado apresentado na Tabela 4.

Tabela 4 - Análise das variáveis de presença de sintomas osteoarticulares e EVA nos idosos do município de Morrinhos, no período de julho a outubro de 2012

\begin{tabular}{lccc}
\hline \multirow{2}{*}{ Presença de sintomas osteoarticulares } & \multicolumn{2}{c}{ EVA } & \\
\cline { 2 - 3 } & Média & Desvio padrão & \\
\hline Sim & 3,96 & 1,6 & $<0,001$ \\
Não & 1,97 & 2,2 & \\
\hline
\end{tabular}

Em relação às alterações decorrentes do processo de envelhecimento, uma das principais queixas dos idosos segundo Andrade, Pereira e Sousa (2006) e Reis et al. (2008) são as alterações osteomioarticulares, geradas por perdas estruturais e funcionais. Segundo Dellaroza, Pimenta e Matsuo (2007) muitos desses quadros são acompanhados por desconfortos álgicos, uma vez que a dor crônica é a principal queixa do indivíduo, podendo interferir de modo acentuado na qualidade de vida dos idosos.

Como mostra a literatura, a doença osteoarticular foi o fator que, mais frequentemente causou dor nos idosos (CUNHA; MAYRINK, 2011).

\section{Conclusão}

Os resultados deste estudo permitem concluir que há uma alta prevalência de alterações osteomioarticulares na população idosa do município de Morrinhos, e que essas, geralmente, são acompanha- das por desconfortos álgicos. Fato que poderá ser agravado pela intensidade dessa experiência, referida como leve a moderada, quando avaliada pela escala análogo visual de dor. Os dados ressaltam, ainda, que essas dores interferem na qualidade de vida, pois, para $46,9 \%$ dos idosos entrevistados, são consideradas um incômodo razoável.

Além dessas conclusões, constatou-se que a relação entre os sintomas osteomioarticulares e a dor apresentou significância estatística.

Com este estudo, espera-se colaborar para a reflexão dos profissionais de saúde e gestores quanto às medidas alternativas existentes para tratamento e manutenção do controle da dor e dos sintomas osteomioarticulares em idosos e, também, sensibilizá-los quanto ao desconforto, sofrimento e incapacidades que podem estar associados a essas condições. 


\section{Correlation of changes and pain in elderly osteomyoarticular of Morrinhos (CE)}

\section{Abstract}

Aging is characterized as a dynamic and progressive process to all living beings, giving each own it ages, resulting characteristics of both morphological, functional and biochemical changes, which will interfere with the individual's ability to adapt to the environment in which lives, causing typical and inevitable functional limitations. Since the increase in the elderly population poses several problems, it is important to analyze the prevalence of musculoskeletal changes, as well as the painful features present in this population. Objective: to correlate skeletal abnormalities and pain in elderly Morrinhos (CE). Methods: cross-sectional, descriptive and analytical study, a quantitative approach, in the city of Morrinhos. The survey was conducted with 270 elderly residents in the municipality. Visual Analogue Scale (Vas) and Nordic Musculoskeletal Questionnaire was used. Data were analyzed using the Spearman correlation test and t-test for independent samples, considering statistical significance of $p \leq 0.05$. Results: among the clinical features, we can highlight: $77.8 \%$ of seniors said osteoarticular symptoms present, as the characterization of pain, $86.5 \%$ reported pain in some part of the body, joint pain being the most present $(81.8 \%)$. One can even say that the presence of Skeletal abnormalities was more related to the occurrence of pain in the elderly factor. Conclusion: we found a high prevalence of musculoskeletal symptoms in the elderly population of the municipality of Morrinhos and these are usually accompanied by nociceptive discomforts.

Keywords: Eldery. Musculoskeletal system. Pain.

\section{Referências}

ANDRADE, F. A. DE; PEREIRA, L. V.; SOUSA, F. A. E. F. Mensuração da dor no idoso: uma revisão. Revista Latino-Americana de Enfermagem, Ribeirão Preto, v. 14, n. 2, p. 271-276, abr. 2006.

BATAGELLO, R. Resolução CNS n. 466, de 12 de dezembro de 2012 - Estabelece as diretrizes e normas brasileiras regulamentadoras de pesquisas envolvendo seres humanos. Revista Brasileira de Bioética, Brasília, v. 8, n. 1-4, p. 105-20, 2012.

BRASIL. Ministério da Saúde - MS. Envelhecimento e saúde da pessoa idosa. 19. ed. Brasília: Ministério da Saúde, 2006, p. 91.

CELICH, K. L. S.; GALON, C. G. Dor crônica em idosos e sua influência nas atividades da vida diária e convivência social. Revista Brasileira de Geriatria e Gerontologia, Rio de Janeiro, v. 12, n. 3, p. 345-359, 2009.

CONTE, E. M. T.; LOPES; SILVA, A. da. Indicadores de qualidade de vida em mulheres idosas. Florianópolis: Universidade Federal de Santa Catarina, 2004.

CUNHA, L. L.; MAYRINK, W. C. Influência da dor crônica na qualidade de vida em idosos. Revista Dor, São Paulo, v. 12, n. 2, p. 120-124, 2011.

DELLAROZA, M. S. G. et al. Caracterização da dor crônica e métodos analgésicos utilizados por idosos da comunidade. Revista da Associação Médica Brasileira, São Paulo, v. 54, n. 1 , p. 36-41, fev. 2008.

DELlAROZA, M. S. G.; PIMENTA, C. A.; MATSUO, T. Prevalência e caracterização da dor crônica em idosos não institucionalizados. Cadernos de Saúde Pública, Rio de Janeiro, v. 23, n. 5, p. 1151-1160, 2007.

FIEDLER, M. M.; PERES, K. G. Capacidade funcional e fatores associados em idosos do Sul do Brasil: um estudo de base populacional. Cadernos de Saúde Pública, Rio de Janeiro, v. 24, n. 2, p. 409-415, 2008a. 
FIEDLER, M. M.; PERES, K. G. Capacidade funcional e fatores associados em idosos do Sul do Brasil: um estudo de base populacional. Cadernos de Saúde Pública, Rio de Janeiro, v. 24, n. 2, p. 409-415, fev. 2008b.

GOMES, R.; NASCIMENTO, E. F.; ARAÚJO, F. Por que os homens buscam menos os serviços de saúde do que as mulheres? As explicações de homens com baixa escolaridade e homens com ensino superior. Cadernos de Saúde Pública, Rio de Janeiro, v. 23, n. 3, p. 565-574, 2007.

GOUVEIA, G. P. de M. Funcionalidade e qualidade de vida como preditores da ocorrência de quedas em idosos do município de Sobral. Fortaleza: Universidade Federal do Ceará, 2011.

JAKOBSSON, U. Pain management among older people in need of help with activities of daily living. Pain management nursing: official journal of the American Society of Pain Management Nurses, Lenexa (KS), v. 5, n. 4, p. 137-43, dez. 2004.

LACERDA, P. F. et al. Estudo da ocorrência de "dor crônica" em idosos de uma comunidade atendida pelo programa saúde da família em Goiânia. Revista Eletrônica de Enfermagem, Goiânia, v. 7, n. 1, p. 29-40, 2005.

LEBRÃO, M. L.; DUARTE, Y. A. O. Saúde, Bem-estar e Envelhecimento - o Projeto Sabe no município de São Paulo: uma abordagem inicial. Brasília: Athalaia Gráfica Ltda, 2003. p. 255.

MAZO, G. Z. Atividade física e qualidade de vida de mulheres idosas. Revista Brasileira de Cineantropometria e desempenho humano, Florianópolis, v. 5, n. 2, p. 87, 2003.

PANAZZOLO, D. et al. Dor crônica em idosos moradores do conjunto cabo frio, cidade de Londrina/PR. Revista Dor, São Paulo, v. 8, n. 3, p. 1052-1054, 2007.

PILGER, C.; MENON, M. H.; MATHIAS, T. A. de F. Socio-demographic and health characteristics of elderly individuals: support for health services. Revista Latino-Americana de Enfermagem, Ribeirão Preto, v. 19, n. 5, p. 1230-1238, out. 2011.

REIS, L. A. dos. et al. Saúde dos idosos da clínica-escola de fisioterapia da universidade estadual do sudoeste da Bahia. Ciência, Cuidado e Saúde, Maringá, v. 7, n. 2, p. 187-192, 2008.

SANTOS, F. C. et al. Programa de autogerenciamento da dor crônica no idoso: estudo piloto. Revista Dor, São Paulo, v. 12, n. 3, p. 209-214, set. 2011.

SCHVEITZER, V.; CLAUDINO, R. A importância da atividade física durante o processo de envelhecimento. Lecturas Educación Física y Deportes, Buenos Aires, v. 14, n. 141, 2010.

SOUZA, L. M. DE; MORAIS, E. P. DE; BARTH, Q. C. M. Socioeconomic and demographic characteristics and health condition of elderly people from a family health program in Porto Alegre, Brazil. Revista Latino-Americana de Enfermagem, Ribeirão Preto, v. 14, n. 6, p. 901-906, dez. 2006.

TSUKIMOTO, G. R. et al. Avaliação longitudinal da Escola de Postura para dor lombar crônica através da aplicação dos questionários Roland Morris e Short Form Health Survey (SF-36). Acta fisiátrica, São Paulo, v. 13, n. 2 , p. $63-69,2006$. 